\title{
In Vivo Formation of $\gamma$-H2AX and 53BP1 DNA Repair Foci in Blood Cells After Radioiodine Therapy of Differentiated Thyroid Cancer
}

Michael Lassmann ${ }^{1}$, Heribert Hänscheid ${ }^{1}$, Daniela Gassen ${ }^{2}$, Johannes Biko ${ }^{1}$, Viktor Meineke ${ }^{2}$, Christoph Reiners ${ }^{1}$, and Harry Scherthan ${ }^{2}$

${ }^{I}$ Department of Nuclear Medicine, University of Würzburg, Würzburg, Germany; and ${ }^{2}$ Bundeswehr Institute of Radiobiology, affiliated to the University of Ulm, Munich, Germany

DNA double-strand breaks (DSBs) are critical cellular lesions that can result from ionizing radiation exposure. A marker for DSB formation is the phosphorylated form of the histone $\mathrm{H} 2$ variant $\mathrm{H} 2 \mathrm{AX}(\gamma-\mathrm{H} 2 \mathrm{AX})$. DSBs also attract the damage sensor p53-binding protein 1 (53BP1) to the DSB-containing chromatin, because 53BP1 associates with the DSB-surrounding chromatin. We studied the induction, persistence, and disappearance of radiation-induced $\gamma-\mathrm{H} 2 \mathrm{AX}$ and 53BP1 foci after the first ${ }^{131}$ I therapy of patients with differentiated thyroid carcinoma, a model for protracted, continuous, internal whole-body irradiation. Methods: Twenty-six patients (7 men, 19 women; mean age $\pm S D, 42 \pm 13$ y) underwent posttherapeutic blood dosimetry according to the standard operating procedure of the European Association of Nuclear Medicine, including peripheral blood sampling and external dose rate measurements at 2-144 $\mathrm{h}$ after administration of ${ }^{131} \mathrm{I}$ for thyroid remnant ablation. The mean time curves of dose accumulation and dose rate to the blood were compared with the mean $\gamma-\mathrm{H} 2 \mathrm{AX}$ and 53BP1 foci counts over the same period in samples of mononuclear peripheral blood leukocytes. Results: The mean absorbed dose to the blood in 24 patients evaluable for physical dosimetry was 0.31 \pm 0.10 Gy (minimum, 0.17 Gy; maximum, 0.57 Gy). After 24 h, the mean daily dose increment was less than 0.05 Gy. The excess focus counts per nucleus - that is, nuclear foci in excess of the low background count-peaked at $2 \mathrm{~h}$ after radioiodine administration (median excess foci for $\gamma$-H2AX $[n=21$ patients], 0.227, and for 53BP1 [ $n=19$ patients], 0.235) and progressively declined thereafter. Significantly elevated numbers of excess focus counts per nucleus (median excess foci for $\gamma-\mathrm{H} 2 \mathrm{AX}[n=8$ patients], 0.054, and for 53BP1 $[n=6$ patients], 0.046) still were present at 120-144 h after therapy. Because the rate of occurrence of radiation-induced focus counts per nucleus per absorbed dose varied considerably among patients, a dose-response relationship could not be established for this series as a whole. The number of excess radiation-induced focus counts per nucleus per absorbed dose

Received Oct. 16, 2009; revision accepted Dec. 2, 2009. For correspondence or reprints contact: Michael Lassmann, Department of Nuclear Medicine, University of Würzburg, Oberdürrbacher Strasse 6, D-97080 Würzburg, Germany.

E-mail: Lassmann_m@klinik.uni-wuerzburg.de

COPYRIGHT ๑ 2010 by the Society of Nuclear Medicine, Inc. rate increased with time, potentially indicating a slower rate of DNA repair or, alternatively, a higher de novo rate of focus formation. The values over time of both radiation-induced DSB markers correlated closely $\left(r^{2}=0.973\right)$. Conclusion: Radiation-induced $\gamma-\mathrm{H} 2 \mathrm{AX}$ and 53BP1 nuclear foci are useful markers for detecting radiation exposure after radionuclide incorporation, even for absorbed doses to the blood below 20 mGy.

Key Words: $\gamma-\mathrm{H} 2 \mathrm{AX}$; 53BP1; biological dosimetry; radionuclide therapy; ${ }^{131}$ I

J Nucl Med 2010; 51:1318-1325

DOI: 10.2967/jnumed.109.071357

\section{$\mathrm{E}$} xposure to ionizing radiation causes several lesions in affected cells because of direct ionization or radical attack (1). Among the most critical defects are DNA double-strand breaks (DSBs), which can lead to efficient cell killing or, through erroneous DNA repair, can be a source of stochastic damage, including increased risk of malignant transformation.

Once a DSB forms, the cell initiates the DNA damage response, to which the ataxia-telangiectasia-mutated (ATM) protein, ataxia-telangiectasia and rad3-related protein, (ATR), and DNA-dependent protein kinase (DNAPKs) are central. An immediate consequence of a DSB is the phosphorylation of Ser139 of the minor histone $\mathrm{H} 2$ variant $\mathrm{H} 2 \mathrm{AX}$ in megabase domains surrounding the DSB - the phosphorylated molecule being termed $\gamma$-H2AX (2). DSBs also attract the damage sensor p53-binding protein 1 (53BP1) to the surrounding chromatin, where the 53BP1 is retained by $\gamma-\mathrm{H} 2 \mathrm{AX}$ and signals chromatin damage (3).

At the microscopic level, the presence of $\gamma$-H2AX or $53 \mathrm{BP} 1$ leads to the formation of nuclear foci, a phenomenon also observed with other proteins and modifications involved in DNA damage repair or signaling, for example, NBS1, ATM-Ser1981-P, or Mre11 (4,5). DSBs cause $\gamma$-H2AX formation within seconds to minutes, and in vitro, 
the yield of nuclear foci shows a linear relationship with radiation dose over a wide dose range $(6,7)$. Therefore, the enumeration of ionizing radiation-induced focus (IRIF) counts per nucleus-that is, nuclear foci in excess of the low background count-has been exploited in biodosimetric settings $(6,7)$. A relationship between the IRIF count and absorbed dose after short-term partial-body irradiation has been established in vivo for CT scans $(8,9)$ and for patients in radiation oncology (10). However, to our knowledge, radiation-induced $\gamma-\mathrm{H} 2 \mathrm{AX}$ and 53BP1 focus formation has not yet been quantified after systemic radionuclide therapy.

After total or near-total thyroidectomy for differentiated thyroid cancer (DTC), patients are commonly treated with one or more courses of ${ }^{131}$ I (radioiodine therapy). The purpose of the first radioiodine therapy after surgery is to ablate remnant thyroid tissue and treat any iodine-avid metastases (11). Patients with DTC generally do not receive chemotherapy or other radiotherapy before radioiodine therapy. Therefore, the administration of large ${ }^{131} \mathrm{I}$ activities provides ideal conditions to assess the effects on leukocytes of prolonged irradiation by radionuclide incorporation and subsequent systemic $\beta$ - and $\gamma$-ionizing radiation exposure. Specifically, this setting provides good opportunities to investigate the DNA damage induced in these cells in vivo after protracted, nearly homogeneous whole-body irradiation.

After radioiodine administration, the absorbed dose and dose rate to the blood are quantifiable by measuring the time-activity curves in the blood and whole body. The blood is irradiated by $\beta$-particles emitted from circulating ${ }^{131} \mathrm{I}$ and from penetrating $\gamma$-radiation originating from activity dispersed throughout the body. A standard operating procedure (SOP) for determining the absorbed dose to the blood was recently published by the Dosimetry Committee of the European Association of Nuclear Medicine (EANM) (12).

In the present study, we investigated the induction, persistence, and turnover of DSB-induced $\gamma-\mathrm{H} 2 \mathrm{AX}$ and 53BP1 nuclear foci after radionuclide incorporation in patients with DTC after their first radioiodine therapy. Additionally, we correlated the radiobiologic findings with data on absorbed doses and dose rates to the blood and tested the suitability of each DNA damage marker as an in vivo biologic dosimeter after prolonged internal wholebody irradiation.

\section{MATERIALS AND METHODS}

\section{Patients}

This study included 26 patients from the University of Würzburg ( 7 men, 19 women; mean age $\pm \mathrm{SD}, 42 \pm 13 \mathrm{y}$; median, $41 \mathrm{y}$; range, 27-70 y) receiving their first radioiodine therapy after surgery for differentiated papillary or follicular thyroid carcinoma; selected patient characteristics are shown in Table 1. No patient had a history of leukemia or lymphoma or radio- or chemotherapy; none underwent a radiographic examina- tion or, other than the remnant tracer uptake measurement, scintigraphy within 3 mo before radioiodine therapy. The study was approved by the University of Würzburg institutional review board, and informed consent was given by each patient.

\section{Radioiodine Administration}

After thyroidectomy and referral to our center, each patient received 3-8 MBq of ${ }^{131}$ I for thyroid bed tracer uptake measurement to screen for thyroid remnants large enough to require reoperation. Patients with a 24-h iodine uptake of more than 5\% in the thyroid bed were excluded. After tracer measurement, 25 of the 26 patients received radioiodine therapy according to EANM guidelines (11), with fixed nominal activities of $3.7 \mathrm{GBq}$ of ${ }^{131} \mathrm{I}$ (actual mean activity, $3.4 \pm 0.3 \mathrm{GBq}$ ). The remaining patient (patient 21 ), in whom metastatic disease was suspected, was given $5.8 \mathrm{GBq}$ of ${ }^{131} \mathrm{I}$. At the time of tracer and therapeutic activity administration, 22 patients were hypothyroid after thyroid hormone withdrawal, and 4 patients (patients 11,21, 22, and 26) were euthyroid and received recombinant human thyroid-stimulating hormone (rhTSH) (Thyrogen; Genzyme Corp.) according to the manufacturer's instructions.

\section{Calculation of Absorbed Dose to Blood}

The absorbed dose to the blood was calculated according to the EANM's SOP for pretherapeutic dosimetry in DTC (12). Briefly, all patients underwent whole-body retention measurements at a nominal 2, 6, and $24 \mathrm{~h}$ after the therapeutic ${ }^{131} \mathrm{I}$ administration and then daily during hospitalization. The patients did not micturate or defecate between radioiodine administration and the 2-h measurement but were asked to empty their urinary bladders before subsequent measurements.

The whole-body measurements were performed as conjugate view (anterior and posterior) counts using a survey meter at a fixed distance of $2 \mathrm{~m}$. The whole-body retention was calculated by normalizing the geometric mean of subsequent backgroundcorrected anterior and posterior counts to the initial (2-h) measurement. Blood samples were taken at the same times as the whole-body measurements were performed. The activity concentrations in these samples were measured in a calibrated well counter using an $\mathrm{NaI}$ detector for $\gamma$-spectroscopy. Data integrity was ensured by application of quality control procedures recommended in the EANM's SOP (12).

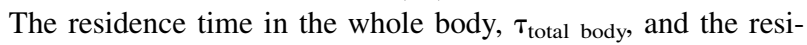
dence time in a milliliter of whole blood, $\tau_{\mathrm{mL}}$ of blood, were determined by integrating biexponential decay curves fitted to the whole-body retention and blood activity concentration data, respectively. The actual blood dose values, $\overline{\mathrm{D}}_{\text {blood }}$ per administered activity $\mathrm{A}_{0}$, were calculated using Equation 1 from the EANM's SOP (12):

$$
\begin{aligned}
\frac{\overline{\mathrm{D}}_{\text {blood }}}{\mathrm{A}_{0}}\left[\frac{\mathrm{Gy}}{\mathrm{GBq}}\right]= & 108 \times \tau_{\mathrm{mL} \text { of blood }}[\mathrm{h}] \\
& +\frac{0.0188}{(\text { weight }[\mathrm{kg}])^{2 / 3}} \times \tau_{\text {total body }}[\mathrm{h}] . \quad \text { Eq. } 1
\end{aligned}
$$

The time curve of the cumulated absorbed dose to the blood, $D_{\text {blood }}(t)$, was deduced by calculating the fraction of the residence times already passed at time $t$ from the biexponential activity curves for each patient. The dose rate was calculated as the derivative of $D_{\text {blood }}(t)$. 
TABLE 1. Selected Patient Characteristics and Dosimetry Results

\begin{tabular}{|c|c|c|c|c|c|}
\hline Patient no. & Sex & $\begin{array}{c}\text { Age at } \\
\text { treatment }(y)\end{array}$ & $\begin{array}{c}\text { Administered } \\
131 \text { I activity }(\mathrm{GBq})\end{array}$ & $\begin{array}{l}\text { Specific absorbed } \\
\text { dose to blood } \\
\text { (mGy/MBq) }\end{array}$ & $\begin{array}{c}\text { Absorbed } \\
\text { dose to blood (Gy) }\end{array}$ \\
\hline 1 & $\mathrm{~F}$ & 29 & 3.6 & 0.133 & 0.48 \\
\hline 2 & $\mathrm{~F}$ & 48 & 3.6 & 0.086 & 0.30 \\
\hline 3 & $\mathrm{M}$ & 47 & 3.6 & 0.069 & 0.25 \\
\hline 4 & $\mathrm{~F}$ & 49 & 3.5 & 0.083 & 0.29 \\
\hline 5 & $\mathrm{~F}$ & 27 & 3.0 & 0.114 & 0.34 \\
\hline 6 & $\mathrm{~F}$ & 43 & 3.7 & 0.156 & 0.57 \\
\hline 7 & $\mathrm{~F}$ & 28 & 3.3 & 0.097 & 0.32 \\
\hline 8 & $\mathrm{M}$ & 50 & 3.6 & 0.065 & 0.23 \\
\hline 9 & $\mathrm{~F}$ & 35 & 3.1 & 0.127 & 0.39 \\
\hline 10 & $\mathrm{~F}$ & 32 & 3.6 & 0.052 & 0.19 \\
\hline $11^{*}$ & $\mathrm{~F}$ & 47 & 3.8 & 0.071 & 0.27 \\
\hline 12 & $\mathrm{~F}$ & 70 & 3.6 & 0.079 & 0.28 \\
\hline 13 & $\mathrm{~F}$ & 35 & 3.5 & NC & $\mathrm{NC}$ \\
\hline 14 & M & 29 & 3.5 & 0.061 & 0.21 \\
\hline 15 & $\mathrm{~F}$ & 54 & 3.7 & 0.048 & 0.17 \\
\hline 16 & $\mathrm{~F}$ & 39 & 3.2 & 0.106 & 0.34 \\
\hline 17 & $\mathrm{~F}$ & 29 & 3.1 & 0.095 & 0.29 \\
\hline 18 & $\mathrm{~F}$ & 25 & 3.4 & 0.081 & 0.28 \\
\hline 19 & $\mathrm{~F}$ & 50 & 2.7 & 0.136 & 0.37 \\
\hline 20 & $\mathrm{~F}$ & 58 & 3.5 & 0.096 & 0.33 \\
\hline $21^{*}$ & $\mathrm{M}$ & 64 & 5.8 & 0.385 & 2.23 \\
\hline $22^{*}$ & $\mathrm{~F}$ & 36 & 3.5 & 0.065 & 0.23 \\
\hline 23 & $\mathrm{M}$ & 38 & 3.4 & 0.050 & 0.17 \\
\hline 24 & $\mathrm{M}$ & 25 & 2.9 & 0.118 & 0.34 \\
\hline 25 & $\mathrm{M}$ & 44 & 3.6 & 0.073 & 0.26 \\
\hline $26^{*}$ & $\mathrm{~F}$ & 68 & 2.9 & 0.180 & 0.52 \\
\hline Mean $\pm \mathrm{SD}^{\dagger}$ & & $42 \pm 13$ & $3.4 \pm 0.3$ & $0.093 \pm 0.035$ & $0.31 \pm 0.10$ \\
\hline Median ${ }^{\dagger}$ & & 41 & 3.4975 & 0.084 & 0.29 \\
\hline \multicolumn{6}{|c|}{$\begin{array}{l}\text { *These patients received radioiodine therapy while euthyroid, after rhTSH administration; all other patients received radioiodine } \\
\text { therapy while hypothyroid, after thyroid hormone withdrawal. } \\
{ }^{+} n=24 \text { for physical dosimetry data; these data do not include patient } 13 \text {, because no blood could be drawn from this individual, or } \\
\text { patient } 21 \text {, because posttherapy whole-body scan revealed multiple highly iodine-avid metastases that resulted in outlier dosimetry } \\
\text { values. } \\
\quad \text { NC }=\text { not calculated. }\end{array}$} \\
\hline
\end{tabular}

Because DNA repair rapidly reduces the number of $\gamma-\mathrm{H} 2 \mathrm{AX}$ and 53BP1 foci after irradiation $(8,13,14)$, we compared the blood dose rate to the number of IRIF to generate dose rate-response curves.

\section{$\gamma$-H2AX and 53BP1 Immunofluorescence}

Leukocyte sample preparation and $\gamma-\mathrm{H} 2 \mathrm{AX}$ and 53BP1 immunostaining and quantification were essentially done as described previously (15), with some modifications. Briefly, after blood collection into cell preparation tubes (Vacutainer CPT; BD), leukocytes were separated by centrifugation at $1,500 \mathrm{~g}$, according to the manufacturer's instructions (BD). Thereafter, leukocytes were recovered above the interphase, washed twice in phosphatebuffered saline (PBS), and after centrifugation, the pellet was mixed with cold $96 \%$ ethanol to result in a final concentration of $3 \times 10^{6}$ cells $/ \mathrm{mL}$ in $70 \%$ ethanol. In this way, the cell preparations were either stored at $-20^{\circ} \mathrm{C}$ or shipped frozen to the Bundeswehr Institute of Radiobiology, Munich, Germany, where all $\gamma-\mathrm{H} 2 \mathrm{AX}$ and 53BP1 focus quantification took place. The storage in EthOH did not affect focus stain ability and yield (Harry Scherthan and Daniela Gassen, unpublished data, 2009).

DSB-associated foci were revealed by immunostaining. To this end, a cell aliquot $\left(3 \times 10^{5}\right)$ was centrifuged on a glass slide
(Superfrost Plus; Menzel Gläser) using a Cytospin 3 cytocentrifuge (Thermo Shandon), fixed for $5 \mathrm{~min}$ in ice-cold $1 \%$ formaldehyde (Merck) in PBS, and rinsed once in PBS/0.1\% glycine and twice in PBS. Cells were extracted at $10 \mathrm{~min}$ in $0.25 \%$ Triton-X-100 (Sigma) in PBS. Then slides were subjected to anti- $\gamma-\mathrm{H} 2 \mathrm{AX}-53 \mathrm{BP} 1 \mathrm{immunofluorescent}$ staining using a mouse monoclonal anti $\gamma$-H2AX antibody (Upstate) at a ratio of 1:500 and a rabbit anti-53BP1 antibody (Acris Antibodies) at a ratio of $1: 250$ in PBS, $0.1 \%$ bovine serum albumin, $0.05 \%$ polysorbate20 , and $0.3 \%$ fish gelatin (PBTG buffer) (16). The preparations were incubated with the antibody solution at $4^{\circ} \mathrm{C}$ overnight, followed by three 5-min washes in PBTG at $37^{\circ} \mathrm{C}$. Primary antibodies were detected by a 45 -min incubation in PBTG at $37^{\circ} \mathrm{C}$ with secondary goat antimouse-Alexa488 (1:500; Mobitec) for anti- $\gamma-\mathrm{H} 2 \mathrm{AX}$ and with secondary donkey antirabbit-Cy3 (1:250; Dianova) for anti-53BP1. Finally, the preparations were subjected to five 3-min washes in PBTG at $37^{\circ} \mathrm{C}$, followed by embedding in Vectashield antifade solution (Vector) containing diamidino-2-phenylindole as a DNA-specific counterstain. Preparations were evaluated using a Zeiss (Jena) Axioplan 2 imaging epifluorescence microscope (equipped with filter blocks for excitation of red, green, and blue fluorescence) and the ISIS Imaging 
System (MetaSystems) (Fig. 1). Granulocytes and monocyteswhich often showed strong autofluorescence-and disrupted cells were excluded from microscopic analysis. For each blood-sampling time point, cell and focus counting was performed until at least 40 cells with at least 1 focus or 800 cells without a focus were registered, whichever occurred first.

The number of $\gamma-\mathrm{H} 2 \mathrm{AX}$ or 53BP1 foci per nucleus was calculated according to the formula $\Sigma_{\text {foci }} / n_{\text {total cells }}$, with $\Sigma_{\text {foci }}$ representing all the foci recorded in the cells counted at a given time point and $n_{\text {total cells }}$ being the total number of cells evaluated at that point. All counting was performed manually by a single operator to minimize variation.

The background frequencies of $\gamma-\mathrm{H} 2 \mathrm{AX}$ or 53BP1 foci in leukocyte nuclei before radioiodine therapy were derived from samples taken immediately before radioiodine therapy.

After activity administration, $\gamma-\mathrm{H} 2 \mathrm{AX}$ and 53BP IRIF were quantified in samples taken at 2 and $24 \mathrm{~h}$ after radioiodine administration and then daily for as long as the patient remained hospitalized, which is at least $48 \mathrm{~h}$ according to the German radiation protection regulations. Usually, one counting of $\gamma$-H2AX and 53BP1 foci was performed per sample; in a few cases, 2 counts were obtained and the values averaged.

\section{Statistics}

Data are expressed as the mean \pm SD for normal distributed data; otherwise, the median and range are given. All statistical tests involving the biomarkers were performed applying the Mann-Whitney (Wilcoxon rank sum) test. A $P$ value less than 0.05 was considered significant. Origin Pro 8G (Origin Lab Corp.) software was used for the tests.

\section{RESULTS}

\section{Absorbed Dose to Blood}

One patient (patient 13) had no blood drawn because of technical difficulties; thus, 25 patients had individual

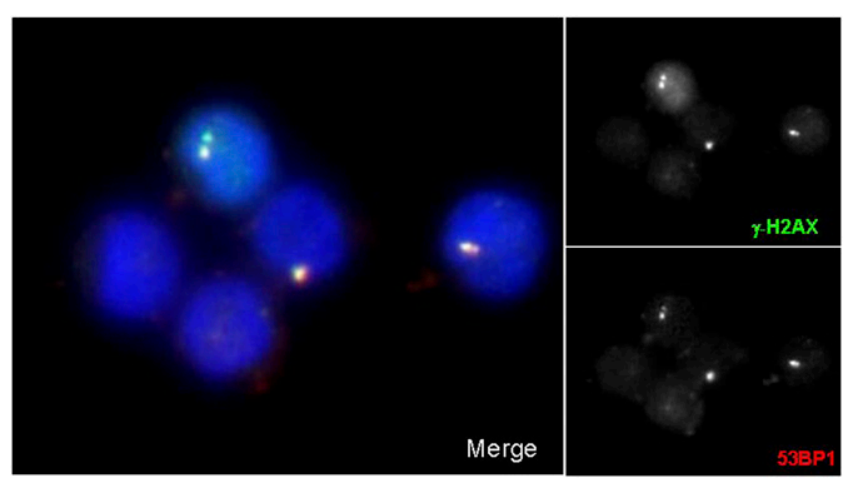

FIGURE 1. Immunofluorescent staining of $\gamma-\mathrm{H} 2 \mathrm{AX}$ (green) and 53BP1 (red) foci in nuclei (blue, DNA) of peripheral blood leukocytes of P22 at $2 \mathrm{~h}$ after administration of $3.5 \mathrm{GBq}$ of ${ }^{131} \mathrm{I}$. Image was taken after $\gamma-\mathrm{H} 2 \mathrm{AX}$ and 53BP1 immunofluorescent staining. Foci for both markers arise at DSB sites, causing signal to appear yellowish because of overlap in merged image. To the right, details of same image separately show green (upper detail, $\gamma-\mathrm{H} 2 \mathrm{AX}$ ) and red (lower detail, 53BP1) channels at $50 \%$ size to display individual foci. physical dosimetry performed (Table 1). Among these patients, patient 21 was excluded from the group analyses after posttherapy whole-body scintigraphy revealed bulky metastases with high radioiodine uptake. Therefore, 24 patients were evaluable for the group physical dosimetry analyses.

The mean specific absorbed dose to the blood in the evaluable patients was $0.093 \pm 0.035 \mathrm{mGy} / \mathrm{MBq}$ (median, $0.084 \mathrm{mGy} / \mathrm{MBq}$ ), resulting in a mean absorbed blood dose of $0.31 \pm 0.10 \mathrm{~Gy}$ (median, $0.29 \mathrm{~Gy}$; minimum, $0.17 \mathrm{~Gy}$; and maximum, $0.57 \mathrm{~Gy}$ ). Twenty-four hours after radioiodine therapy, the mean absorbed dose to the blood was $0.18 \pm 0.04 \mathrm{~Gy}$; for subsequent days, the daily mean dose increment was less than $0.05 \mathrm{~Gy}$. The mean absorbed dose rate was $15.4 \pm 3.2 \mathrm{mGy} / \mathrm{h}$ at $2 \mathrm{~h}, 3.4 \pm 0.9 \mathrm{mGy} / \mathrm{h}$ at $24 \mathrm{~h}$, $1.1 \pm 0.4 \mathrm{mGy} / \mathrm{h}$ at $48 \mathrm{~h}, 0.8 \pm 0.3 \mathrm{mGy} / \mathrm{h}$ at $72 \mathrm{~h}$, and less than $0.5 \mathrm{mGy} / \mathrm{h}$ at $96 \mathrm{~h}$ and later.

Figure 2 shows representative time functions of the activities in the total body and activities per liter of blood (Fig. 2A) and the resulting blood cumulative absorbed dose and dose rate (Fig. 2B) for patient 4 , with an absorbed dose to the blood identical to the median value $(0.29 \mathrm{~Gy})$. The dose rate decreased with time, corresponding to the disappearance of ${ }^{131}$ I activities from the organism. Values at posttherapy times earlier than the first measurement at a nominal $2 \mathrm{~h}$ were uncertain and were not calculated.

\section{$\gamma$-H2AX and 53BP1 Focus Yield}

Table 2 gives the median, minimum, and maximum preradioiodine therapy $\gamma$-H2AX and 53BP1 focus counts per nucleus (background) and corresponding IRIF counts per nucleus - that is, the postradioiodine administration $\gamma$-H2AX and 53BP1 focus counts per nucleus corrected for the pretherapy counts per nucleus-for each posttherapy blood-sampling time point. The numbers of evaluable patients vary for each focus type and measurement time point because of immunostaining failure in all posttherapy samples ( $n=3$, patients $1-3), 53$ PB1 immunostaining failure in all posttherapy samples $(n=2$, patients 9 and 16), unavailability of blood samples for any time point $(n=1$, patient 13), or unavailability of samples at particular posttherapy time points because of technical or logistical problems, such as vein collapse or early discharge from the hospital. The reasons for the immunostaining failures are unknown but may have been due to sample-handling issues.

The radiation absorbed dose induced excess $\gamma-\mathrm{H} 2 \mathrm{AX}$ and 53BP1 foci in all patients. For both foci types, the numbers of IRIF per nucleus were consistently highest at $2 \mathrm{~h}$ after therapy administration, whereas focus counts per nucleus decreased in parallel with the dose rate. Slightly but statistically significantly elevated $\gamma-\mathrm{H} 2 \mathrm{AX}$ and 53BP1 levels were still present at 120-144 h after therapy (Table 2; Fig. 3).

Within individual patients, the extent of observed IRIF formation did not correspond with the magnitude of the absorbed dose to the blood. For example, the patient with the highest observed numbers of IRIF (patient 4; Fig. 3) had 

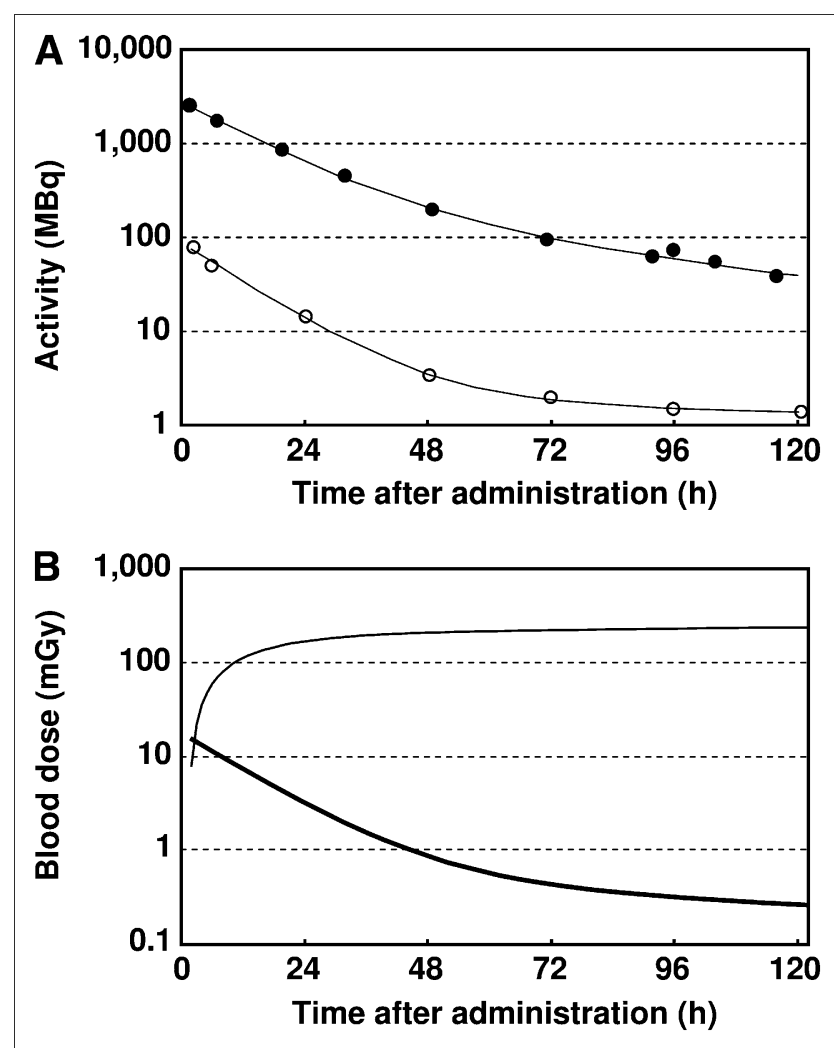

FIGURE 2. Time curves (on logarithmic scale) of representative patient (patient 4 ) with absorbed dose to blood identical to median value of 0.29 Gy. (A) Measured activities and corresponding biexponential fits in whole body (O) and per liter of blood (O). (B) Cumulative absorbed dose to blood (upper curve) and absorbed dose rate (lower bold curve). This patient had highest observed numbers of $\gamma$-H2AX and 53BP1 IRIF.

a blood absorbed dose equal to the median (Table 1). Patient 21 displayed IRIF values within the range for all other patients (Fig. 3), whereas the absorbed blood dose in this individual exceeded that of all other patients by a factor of more than 4 (Table 1). In patient 22, $\gamma$-H2AX IRIF numbers were consistently far lower than those of all other patients (Fig. 3) (double-checked by independent recounting of several samples), while 4 patients had lower blood absorbed doses and another patient had the same blood absorbed dose (Table 1). The reason for the effects in patient 22 is unknown. Speculatively, there could, for instance, be a weaker response to DSB seeding agents in this patient's cells.

Supplemental Figure 1 (available online only at http:// jnm.snmjournals.org) shows the number of $\gamma-\mathrm{H} 2 \mathrm{AX}$ and 53PB1 IRIF, compared with the blood dose rate, as a function of time after ${ }^{131} \mathrm{I}$ administration for all patients, with complete sets of data for at least 5 times $(n=9$; patient 21 was excluded from this comparison because of outlier physical dosimetry data). The numbers of excess foci per dose rate show a high variability, as can be seen from the differing distances between the curves of the dose rate and focus count in the supplemental figure, for instance, when comparing patients 4 and 25 . At early times, the distance between these curves was less for patient 4 , indicating induction of more foci per absorbed dose than in patient 25. The more gradual temporal decrease of both IRIF frequencies in patient 4 than in patient 25 may reflect a slower DNA rate repair or, alternatively (although less likely), a higher de novo rate of focus formation in patient 4 .

The numbers of excess foci seemed to depend on the dose rate-that is, the blood dose absorbed within a short period preceding the sampling for the IRIF counting. However, for most patients, the dose rate declined more steeply over time than did the observed number of excess foci. Thus, the median ratios of IRIF per absorbed dose rate (IRIF per $\mathrm{mGy} / \mathrm{h}$ ) increased significantly from 2 to $24 \mathrm{~h}$ and from 24 to $48 \mathrm{~h}$ (Table 3). Further but statistically not significant $(P>0.05)$ increases in this ratio were observed at later times (data not shown).

In the radioiodine therapy setting, both tests $(\gamma-\mathrm{H} 2 \mathrm{AX}$ and 53BP1) provided almost identical numbers of excess foci, which is also reflected by the overlap of both types of DSB responses in the nucleus (Fig. 1). A comparison of all blood samples evaluated with both tests (Fig. 4) revealed a linear regression that, when forced to intersect the origin, yielded a slope of $1.004 \pm 0.018$ and a correlation coefficient of $r^{2}=0.973$.

\section{DISCUSSION}

The absorbed doses to the blood after ${ }^{131}$ I therapy of patients with DTC in this study agree well with published data from pre- and peritherapeutic settings $(11,17,18)$. Although the radioiodine biokinetics in euthyroid patients after rhTSH administration generally differ from those of hypothyroid patients after thyroid hormone withholding (18), the data of the 3 rhTSH patients included in these group analyses were within the range of the blood doses of the hypothyroid patients and thus did not have to be analyzed separately.

In the present study, the dosimetry data were correlated with a systematic analysis of $\gamma-\mathrm{H} 2 \mathrm{AX}$ and 53BP1 IRIF after systemic in vivo irradiation with ${ }^{131} \mathrm{I}$. By choosing this particular group of patients, who did not receive prior radio- or chemotherapy, influences of medical pretreatments on the biomarkers could be excluded. Additionally, for several reasons, the pretherapeutic tracer activity was extremely unlikely to influence the results of this study. First, because of minimal tracer activity, the absorbed dose to the blood was more than 2 orders of magnitude lower than that of the therapeutic activity (data not shown). Second, because of the rapid disappearance of IRIF (7), the interval of several days between tracer and therapy administration should have more than sufficed to allow the nuclear focus counts to return to background levels. Last, the background values in the present study agreed well with 
TABLE 2. $\gamma$-H2AX and 53BP1 Focus Counts per Nucleus Before and After Radioiodine Therapy

\begin{tabular}{|c|c|c|c|c|c|c|c|}
\hline \multirow[b]{2}{*}{ Variable } & \multirow{2}{*}{$\begin{array}{c}\text { Background } \\
\text { (pretherapy) value }\end{array}$} & \multicolumn{6}{|c|}{ Time after radioiodine administration $(\mathrm{h})$} \\
\hline & & 2 & 24 & 48 & 72 & 96 & $120-144$ \\
\hline \multicolumn{8}{|l|}{$\gamma-\mathrm{H} 2 \mathrm{AX}$} \\
\hline Median & 0.0030 & 0.227 & 0.116 & 0.101 & 0.086 & 0.053 & 0.054 \\
\hline Minimum & 0.0012 & 0.026 & 0.016 & 0.010 & 0.001 & 0.005 & 0.021 \\
\hline Maximum & 0.0176 & 0.748 & 0.423 & 0.160 & 0.233 & 0.224 & 0.103 \\
\hline$n^{*}$ & 25 & 21 & 14 & 20 & 18 & 14 & 8 \\
\hline \multicolumn{8}{|l|}{ 53BP1 } \\
\hline Median & 0.0073 & 0.235 & 0.079 & 0.086 & 0.068 & 0.046 & 0.046 \\
\hline Minimum & 0.0012 & 0.037 & 0.041 & 0.027 & 0.014 & 0.004 & 0.019 \\
\hline Maximum & 0.0333 & 0.715 & 0.418 & 0.174 & 0.277 & 0.241 & 0.076 \\
\hline$n^{\star}$ & 23 & 19 & 13 & 19 & 17 & 13 & 6 \\
\hline
\end{tabular}

previously published data from 2 volunteers ((15) investigated at the Bundeswehr Institute of Radiobiology).

In our patient group, we compared 2 separate markers of DNA DSB formation, $\gamma-\mathrm{H} 2 \mathrm{AX}$ and 53BP1, in vivo in patients with radionuclide incorporation. Because the $\gamma$-H2AX test uses a DSB-induced protein modification and 53BP1 foci represent accumulation of a DSB-modified chromatin protein $(3,19,20)$, it appears that the IRIF most likely arose from protracted irradiation of cells by the remaining ${ }^{131}$ I activity in the body. In line with this reasoning, both types of IRIF were nearly identical in median number at all measurement times (Table 2) and foci were physically overlapping in fluorescence images (Fig. 1). The magnitude of IRIF induction generally corresponded to the increase in the absorbed blood dose- that is, the dose rate to the blood or the blood dose absorbed within a short period before sample collection. Elevated levels of IRIF also could be detected at late time points, a finding that cannot be explained quantitatively by the decreased dose rate at that time, but may, for instance, involve bystander effects.
For most patients, the decline of the dose rate-time function was steeper than the observed decrease of excess foci, and the median ratios of IRIF per dose rate significantly increased as a function of time after radioiodine administration. These phenomena suggest the presence of a longlived proportion of foci accumulating with the total absorbed dose, possibly because of the chronic irradiation from incorporated ionizing radiation sources. Because DNA damage by $\beta$-emitters such as ${ }^{131} \mathrm{I}$ seems to arise largely through indirect or radical effects (21), persistent IRIF also could reflect the accumulation of a subset of complex DSBs that are more difficult to repair with time. Furthermore, increasing IRIF rates with time may reflect bystander effects, as low-dose irradiation can induce DSBs in nonirradiated cells through signaling molecules and reactive oxygen species (22). Moreover, in the current low-dose scenario there may be inefficient detection of DNA damage at early time points, leading to damage accumulation before a biologic response is triggered, which would align with in vitro data suggesting that low-dose irradiation may lead to inefficient ATM activation (23).

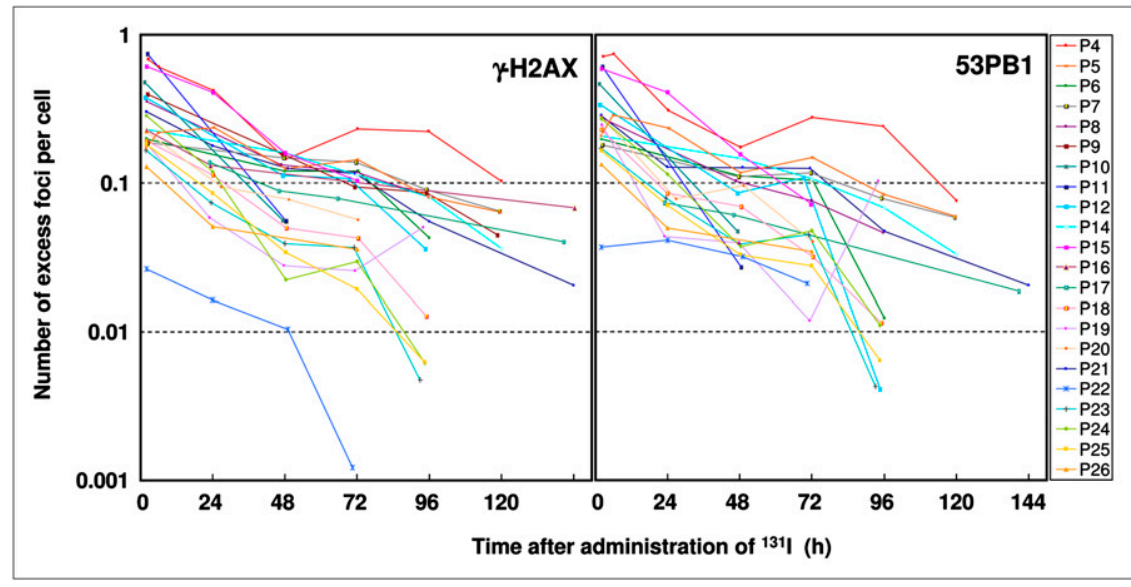

FIGURE 3. Numbers of $\gamma-\mathrm{H} 2 \mathrm{AX}$ or 53BP1 IRIF per leukocyte for each patient as function of time after 131। administration (logarithmic scale). 
TABLE 3. $\gamma$-H2AX and 53BP1 IRIF per Absorbed Dose Rate

\begin{tabular}{|c|c|c|c|c|c|c|}
\hline \multirow[b]{2}{*}{ Variable } & \multicolumn{6}{|c|}{ Time after radioiodine administration (h) } \\
\hline & 2 & 24 & 48 & 72 & 96 & 120 \\
\hline $\begin{array}{l}\gamma-\mathrm{H} 2 \mathrm{AX} \\
\text { Median (mGy/h) } \\
n^{\star}\end{array}$ & $\begin{array}{c}0.0168 \\
20\end{array}$ & $\begin{array}{c}0.0338 \\
13\end{array}$ & $\begin{array}{c}0.0695 \\
19\end{array}$ & $\begin{array}{c}0.108 \\
17\end{array}$ & $\begin{array}{c}0.1413 \\
13\end{array}$ & $\begin{array}{c}0.2764 \\
5\end{array}$ \\
\hline $\begin{array}{l}\text { 53BP1 } \\
\text { Median (mGy/h) } \\
n^{\star}\end{array}$ & $\begin{array}{c}0.0180 \\
20\end{array}$ & $\begin{array}{c}0.0234 \\
13\end{array}$ & $\begin{array}{c}0.0675 \\
19\end{array}$ & $\begin{array}{c}0.1351 \\
17\end{array}$ & $\begin{array}{c}0.1096 \\
13\end{array}$ & $\begin{array}{c}0.1752 \\
5\end{array}$ \\
\hline
\end{tabular}

Further analysis with more patients is needed to elucidate these findings.

A general correlation between absorbed dose or the absorbed dose rate and number of excess foci, however, could not be established because of a large interindividual variability in IRIF response.

The usefulness of the $\gamma-\mathrm{H} 2 \mathrm{AX}$ focus test as a nonpatient-specific biodosimetric test for radiation exposure has also been shown by Löbrich et al. (8) and Rothkamm et al. (9). They observed a good overall correlation of $\gamma$-H2AX focus number and the dose delivered by CT examinations when the blood samples were obtained up to $30 \mathrm{~min}$ after exposure $(8,9)$.

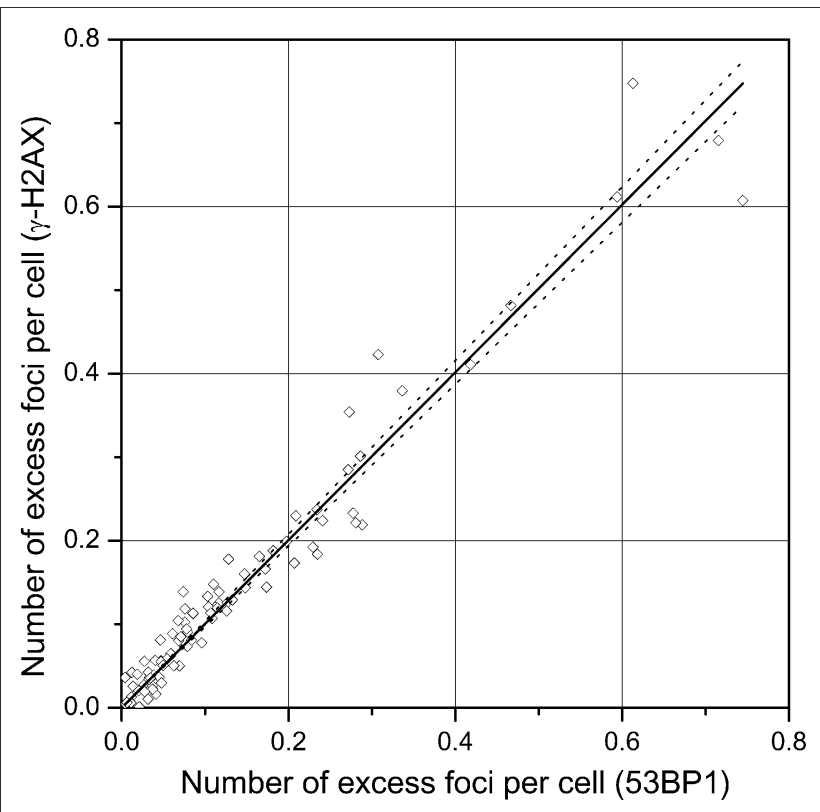

FIGURE 4. Number of $\gamma-\mathrm{H} 2 \mathrm{AX}$ IRIF per cell as function of number of 53BP1 IRIF per cell for all pre- and posttherapy samples evaluable for both markers. Straight line denotes linear correlation $\left(r^{2}=0.973\right) .95 \%$ confidence interval of regression line is plotted with dotted lines.
Our results, however, are not directly comparable to those of Löbrich et al. (8), Rothkamm et al. (9), Sak et al. (10), or Kuefner et al. (24), because the settings differed considerably in several respects. First, those studies administered short-term, external, high-dose-rate irradiation via $\mathrm{CT}$, a linear accelerator, or an angiography system. In those cases, DSBs and DNA repair were triggered by an acute event, and the $\gamma-\mathrm{H} 2 \mathrm{AX}$ measurements were performed immediately after irradiation. Second, with CT, externalbeam therapy, or angiography, there is partial-body irradiation. Presently, no standardized method exists to assess accurately the absorbed dose to the irradiated leukocytes after partial-body exposure because only a fraction of blood cells is exposed to irradiation.

In the case of systemic application of ${ }^{131} \mathrm{I}$, there is a protracted continuous irradiation of the leukocytes and a constantly diminishing dose rate over time, whereas individual patients are likely to have variable efficacy in their DNA repair mechanisms. Thus, the use of the dose rate representing the differential increment of the absorbed dose is an approximation of the actual energy imparted to the leukocytes. However, the situation in the tumor tissue targeted by this treatment will be quite different.

Andrievski and Wilkins (14) assessed the use of $\gamma$-H2AX foci in human blood lymphocytes as a rapid screening tool for radiation biodosimetry. These investigators exposed human peripheral blood to 0-10 Gy of ${ }^{137} \mathrm{Cs}$ irradiation and analyzed $\gamma-\mathrm{H} 2 \mathrm{AX}$ focus formation and turnover in different leukocyte subpopulations. They found that the leukocyte subsets displayed a similar linear dose-response relationship; histone $\mathrm{H} 2 \mathrm{AX}$ phosphorylation of all leukocyte subsets reached a maximum at 1.5 $\mathrm{h}$ and essentially returned to background levels at $24 \mathrm{~h}$ after exposure. Andrievski and Wilkins (14) concluded that the usefulness of $\gamma-\mathrm{H} 2 \mathrm{AX}$ for radiation biodosimetry was hampered by the marker's relatively short life span and the large interindividual variation in the magnitude of marker induction. These findings (14) agree with our results, which show a good correlation of dose rate and focus count and also a substantial interindividual variation. In our case, because the dose resulted from internalized 
radionuclides, the rapid loss of DSB-associated foci that usually occurs after a single acute exposure was likely counteracted by IRIF formation that was driven by de novo DSBs caused by the chronic irradiation by the remaining circulating ${ }^{131} \mathrm{I}$.

Our observations and previous results $(8,9,14)$ suggest that $\gamma$-H2AX is a suitable qualitative biodosimeter, provided samples are processed shortly after exposure. On the basis of the present study, we suggest that for the $\gamma-\mathrm{H} 2 \mathrm{AX}$ test, the median value of our last (120-144 h) time-rounded conservatively to 0.06 focus counts per nucleus - may be used as a cutoff for the detection of internal homogeneous radiation exposure. The corresponding 24-h dose increment 120-144 h after administration of ${ }^{131} \mathrm{I}$ was $20 \mathrm{mGy}$ and lower.

Overall, we consider $\gamma-\mathrm{H} 2 \mathrm{AX}$ and 53BP1 focus tests to be well suited for the detection of radiation exposure after radionuclide incorporation, even in low-dose and low-doserate scenarios, which corroborates earlier in vitro data (7). The focus tests are less well suited for patient-specific dosimetry, because of the high interpatient variability in focus induction, at least as observed in this work.

More in vivo research is needed to better understand and interpret the results of the present study. It will be interesting to obtain further data on the intrapatient reproducibility of the tests after subsequent radioiodine treatments and to learn about the effects of other radionuclides showing different biokinetics, biodistributions, and dose rates.

\section{CONCLUSION}

In this setting of protracted internal whole-body irradiation, the $\gamma$-H2AX and 53BP1 focus tests disclosed a high number of IRIF shortly (at $2 \mathrm{~h}$ ) after radioiodine therapy administration. The focus count subsequently decreased over time but tended to remain elevated above baseline levels for at least $144 \mathrm{~h}$ after therapy. The magnitude of excess focus induction varied markedly between individuals, a finding that typically reflects a low-dose-rate situation. Hence, a reliable dose-response relationship between the absorbed dose (rate) and number of induced foci could be established only within individual patients rather than across a group of patients. Nonetheless, the $\gamma$-H2AX and 53BP1 IRIF quantification appears to be well suited for the detection of radiation exposure after radionuclide incorporation, even in low-dose scenarios in which the absorbed dose to the blood is $20 \mathrm{mGy}$ or less.

\section{ACKNOWLEDGMENTS}

We thank Tanja Inderwies for valuable technical assistance and gratefully acknowledge the invaluable help of Maria Hategan, Frederik A. Verburg, and Kai Nerlich in patient recruitment. The editorial comments of Rob Marlowe are gratefully acknowledged. This work was supported by a contract research project for the Bundeswehr Medical Service.

\section{REFERENCES}

1. Riley PA. Free radicals in biology: oxidative stress and the effects of ionizing radiation. Int J Radiat Biol. 1994;65:27-33.

2. Rogakou EP, Pilch DR, Orr AH, Ivanova VS, Bonner WM. DNA doublestranded breaks induce histone H2AX phosphorylation on serine 139. J Biol Chem. 1998;273:5858-5868.

3. Huyen Y, Zgheib O, Ditullio RA Jr., et al. Methylated lysine 79 of histone H3 targets 53BP1 to DNA double-strand breaks. Nature. 2004;432:406-411.

4. Bekker-Jensen S, Lukas C, Kitagawa R, et al. Spatial organization of the mammalian genome surveillance machinery in response to DNA strand breaks. J Cell Biol. 2006;173:195-206.

5. Keogh MC, Kim JA, Downey M, et al. A phosphatase complex that dephosphorylates gammaH2 AX regulates DNA damage checkpoint recovery. Nature. 2006;439:497-501.

6. Rogakou EP, Boon C, Redon C, Bonner WM. Megabase chromatin domains involved in DNA double-strand breaks in vivo. J Cell Biol. 1999;146:905-916.

7. Rothkamm K, Lobrich M. Evidence for a lack of DNA double-strand break repair in human cells exposed to very low x-ray doses. Proc Natl Acad Sci USA. 2003;100:5057-5062.

8. Löbrich M, Rief N, Kuhne M, et al. In vivo formation and repair of DNA doublestrand breaks after computed tomography examinations. Proc Natl Acad Sci USA. 2005;102:8984-8989.

9. Rothkamm K, Balroop S, Shekhdar J, Fernie P, Goh V. Leukocyte DNA damage after multi-detector row CT: a quantitative biomarker of low-level radiation exposure. Radiology. 2007;242:244-251.

10. Sak A, Grehl S, Erichsen P, et al. Gamma-H2AX foci formation in peripheral blood lymphocytes of tumor patients after local radiotherapy to different sites of the body: dependence on the dose-distribution, irradiated site and time from start of treatment. Int J Radiat Biol. 2007;83:639-652.

11. Luster M, Clarke SE, Dietlein M, et al. Guidelines for radioiodine therapy of differentiated thyroid cancer. Eur J Nucl Med Mol Imaging. 2008;35:1941-1959.

12. Lassmann M, Hänscheid H, Chiesa C, Hindorf C, Flux G, Luster M. EANM Dosimetry Committee series on standard operational procedures for pretherapeutic dosimetry I: blood and bone marrow dosimetry in differentiated thyroid cancer therapy. Eur J Nucl Med Mol Imaging. 2008;35:1405-1412.

13. Olive PL, Banath JP. Phosphorylation of histone H2AX as a measure of radiosensitivity. Int J Radiat Oncol Biol Phys. 2004;58:331-335.

14. Andrievski A, Wilkins RC. The response of gamma-H2AX in human lymphocytes and lymphocytes subsets measured in whole blood cultures. Int J Radiat Biol. 2009;85:369-376.

15. Scherthan H, Hieber L, Braselmann H, Meineke V, Zitzelsberger H. Accumulation of DSBs in gamma-H2AX domains fuel chromosomal aberrations. Biochem Biophys Res Commun. 2008;371:694-697.

16. Scherthan H, Jerratsch M, Li B, et al. Mammalian meiotic telomeres: protein composition and redistribution in relation to nuclear pores. Mol Biol Cell. 2000;11:4189-4203.

17. Hänscheid H, Lassmann M, Luster M, Kloos R, Reiners C. Blood dosimetry from a single measurement of the whole body radioiodine retention in patients with differentiated thyroid carcinoma. Endocr Relat Cancer. 2009;16:1283-1289.

18. Hänscheid H, Lassmann M, Luster M, et al. Iodine biokinetics and dosimetry in radioiodine therapy of thyroid cancer: procedures and results of a prospective international controlled study of ablation after rhTSH or hormone withdrawal. J Nucl Med. 2006;47:648-654.

19. Mochan TA, Venere M, DiTullio RA Jr., Halazonetis TD. 53BP1, an activator of ATM in response to DNA damage. DNA Repair (Amst). 2004;3:945-952.

20. Zgheib O, Pataky K, Brugger J, Halazonetis TD. An oligomerized 53BP1 tudor domain suffices for recognition of DNA double-strand breaks. Mol Cell Biol. 2009;29:1050-1058.

21. Bishayee A, Rao DV, Bouchet LG, Bolch WE, Howell RW. Protection by DMSO against cell death caused by intracellularly localized iodine-125, iodine-131 and polonium-210. Radiat Res. 2000;153:416-427.

22. Han W, Wu L, Hu B, et al. The early and initiation processes of radiationinduced bystander effects involved in the induction of DNA double strand breaks in non-irradiated cultures. Br J Radiol. 2007;80:S7-S12.

23. Collis SJ, Schwaninger JM, Ntambi AJ, et al. Evasion of early cellular response mechanisms following low level radiation-induced DNA damage. J Biol Chem. 2004;279:49624-49632.

24. Kuefner MA, Grudzenski S, Schwab SA, et al. X-ray-induced DNA doublestrand breaks after angiographic examinations of different anatomic regions [in German]. Rofo. 2009;181:374-380. 